\title{
Uma proposta de modelagem matemática para o Problema de Roteamento em Arcos Capacitado e Periódico adaptado
}

\author{
Guilherme V. Batista, Cassius T. Scarpin, \\ Programa de Pós-graduação em Métodos Numéricos em Engenharia, UFPR \\ CESEC - Centro Politécnico \\ 81531-980, Curitiba, PR \\ E-mail: guivbatista89@gmail.com, cassists@gmail.com
}

\begin{abstract}
Resumo: Os problemas de roteamento em arcos têm grande aplicabilidade a problemas reais e podem ser abordados de diversas formas dependendo de suas restrições. Eles consistem em determinar rotas para veículos de forma que atendam um conjunto de arcos em um grafo. $O$ objetivo desse artigo é apresentar uma modelagem matemática para uma adaptação do Problema de Roteamento em Arcos Capacitado e Periódico. Através de programação linear binária pretende-se determinar uma rota para múltiplos veículos respeitando suas capacidades, considerando um horizonte discreto de tempo e que atenda às demandas de cada arco.
\end{abstract}

\section{Introdução}

Muitos problemas logísticos, como localização de facilidades, gerenciamento de armazéns e roteamento de veículos, têm sido resolvidos por pesquisadores. Os quais têm tomado uma sequência de resolução desde decisões estratégicas até operacionais [5]. Um desses problemas é o de roteamento em arcos, em inglês Arc Routing Problem (ARP), que tem por objetivo determinar o menor custo para atravessar um conjunto de arcos de um grafo, com ou sem restrições. Essa classe de problemas pode ser aplicada numa série de contextos práticos como coleta de lixo, entrega de cartas, remoção de neve, inspeção em linhas de energia elétrica, inspeção e manutenção de ferrovias e rodovias.

Ao contextualizar esses problemas obtêm-se algumas variações, uma delas é abordada neste artigo que tem por objetivo propor uma modelagem matemática a partir de um problema real envolvendo uma malha ferroviária. Essa modelagem leva em conta: a capacidade dos veículos em executar o trabalho, o horizonte de tempo em que toda a malha deve ser atendida e a demanda de cada trecho. O problema que envolve todos esses aspectos é conhecido como Problema do Roteamento em Arcos Capacitado e Periódico, em inglês Periodic Capacitated Arc Routing Problem (PCARP), e dependendo da forma como é tratado pode incluir uma série de complicações para sua resolução.

\section{Revisão da literatura}

Segundo Ghiani et al. [4], diferentemente dos problemas de roteamento em nós que são os problemas de roteamento mais estudados, a literatura do problema de roteamento em arcos é mais pobre e desorganizada. Porém, são problemas que vem ganhando cada vez mais importância. De maneira geral, são problemas definidos em um grafo $G=(V, A)$, onde $V$ é o conjunto de vértices (ou nós) e $A$ o conjunto de Arcos que podem ser direcionados ou não, dependendo do problema.

Eiselt et al. [2,3] abordam diversos problemas de ARP, e fazem uma revisão dos principais problemas estudados até o ano de publicação. O primeiro problema encontrado na literatura é o das pontes de Königsberg, no qual é necessário determinar um caminho mínimo em que um indivíduo atravesse todas as 7 pontes que chegam a cidade exatamente uma vez e volte ao seu ponto de origem. O Problema do Carteiro Chinês (Chinese Postman Problem - CPP) é muito parecido com o problema das pontes: um carteiro deve cobrir toda sua rota com possibilidade de atravessar os arcos mais de uma vez. Outro problema de grande aplicabilidade é o Problema do Carteiro Rural (Rural Postman Problemn - RPP), onde é necessário atravessar apenas um subconjunto de $\operatorname{arcos} R \subseteq A$. 
Um RPP pode ser tratado como um Problema de Roteamento em Arcos Capacitado (Capacitated Arc Routing Problem - CARP), o CARP é um problema mais difícil que os já citados, dado que além de simplesmente fazer o roteamento nos arcos, cada arco tem uma determinada demanda e a capacidade de cada veículo ao atender esses arcos deve ser levada em conta. Grande parte dos problemas reais são formulados dessa forma [5].

Uma extensão natural do CARP é o Problema de Roteamento em Arcos Capacitado e Periódico, ao invés do problema ser resolvido para apenas um dia ele é expandido para múltiplos períodos [8]. São problemas que costumam ter um depósito onde devem iniciar e terminar sua rota diariamente.

Lacomme \& Prins [7] propõe uma definição básica para o PCARP, bem como alguns algoritmos para sua resolução. Dado um grafo e um discreto horizonte de tempo $H$ com $n p$ períodos, cada arco tem uma frequência $f(a)$ que pode ser de no máximo uma vez por dia, restrição essa que ainda pode ser expressa com base no intervalo entre serviços no mesmo arco. O mesmo autor ainda afirma: "o PCARP implica na determinação simultânea das decisões no nível tático e operacional em todo horizonte de tempo" (página 539).

Em muitas aplicações o PCARP tem particularidades que o tornam mais complexo, como demandas flutuantes ao longo do tempo ou ainda restrições de intervalos entre dias de serviço (periodicidades ou frequências). Baseado nisso o problema foi classificado em duas classes [6,7] como ilustra a Figura 1.

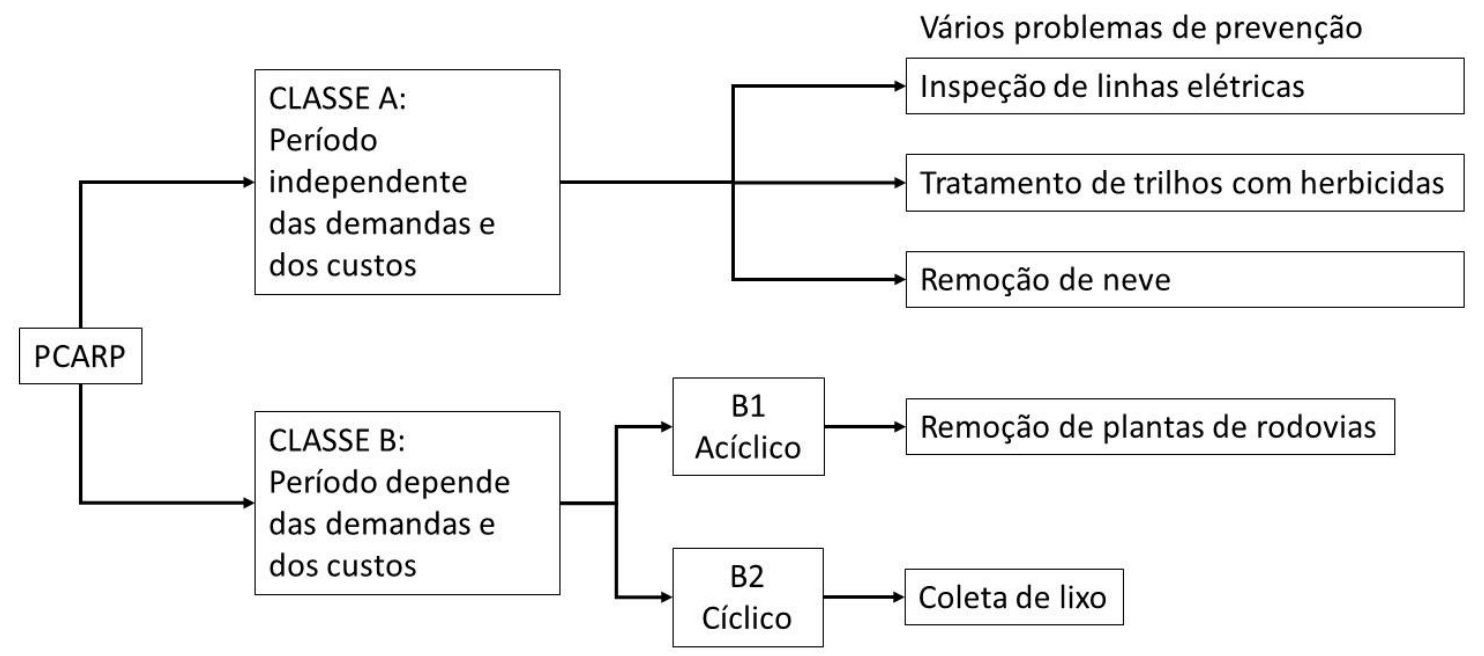

Figura 1: Uma simples classificação do PCARP. Fonte: Lacomme \& Prins [7].

A CLASSE A representa o conjunto dos problemas em que a demanda não resulta de acumulação diária de algum fator, por exemplo, muitos serviços de prevenção, inspeção e monitoramento, como o serviço de pulverização de herbicidas em trilhos de trem onde o tempo gasto por metro e a demanda ao longo do tempo é fixa. Já a CLASSE B envolve problemas como a coleta de lixo, onde a demanda aumenta conforme vão se passando os dias. Além disso, a CLASSE B pode ser subdivida em problemas acíclicos (subclasse B1) ou cíclicos (subclasse B2). Em B1, o horizonte de tempo é bem definido e a demanda é flutuante ao longo do tempo, como no caso da remoção de plantas em rodovias, assumindo que no inverno as plantas não crescem de forma que a demanda é nula. Em B2 o intervalo de tempo se repete continuamente, por exemplo, a coleta de lixo se repete de semana em semana [7].

Chu, Labadi, \& Prins [1], foram os primeiros a propor uma modelagem matemática para o PCARP. Eles conseguiram resultados ótimos em softwares comerciais apenas para instâncias muito pequenas com 5 períodos e 10 arcos, por exemplo. Os autores já esperavam esses resultados devido à natureza do problema - NP-Hard - e por isso propuseram três heurísticas capazes de resolver o problema.

Monroy et al. [8] modela matematicamente o problema de monitoramento da malha rodoviária, que é realizado periodicamente, e propõe uma heurística para sua resolução. As ruas ou rodovias são divididas em classes de acordo com a necessidade de vigilância e a hierarquia das vias. Cada categoria tem sua demanda (número de passagens) durante um horizonte de tempo 
já definido. O objetivo é designar um conjunto de rotas satisfazendo as frequências de cada classe de vias em cada subperíodo de tempo sem exceder a capacidade do veículo.

\section{0 problema}

Um processo que ocorre nas empresas que trabalham com ferrovias é a manutenção preventiva e inspeção dos trilhos. Toda malha ferroviária deve ser avaliada para que sejam prevenidos acidentes, como tombamento de trens. Para isso existem sofisticados equipamentos capazes de fazer medições específicas para prevenir acidentes. Esses equipamentos costumam ter um custo elevado e são como carros - daqui para frente chamaremos esse equipamento de carro - que se deslocam pelos trilhos executando sua tarefa.

A malha ferroviária brasileira tem grande extensão e os equipamentos disponíveis devem ser colocados em locais estratégicos para fazer essas medições, cada trecho tem uma demanda conhecida a priori que diz de quanto em quanto tempo ele deve ser analisado, ou seja, sua periodicidade. O horizonte de tempo se repete formando ciclos, desta forma, faz-se necessário tomar decisões operacionais sobre quais trechos devem ser trabalhados diariamente e, também, decisões estratégicas que abranjam todo o horizonte para evitar atrasos nas periodicidades.

É um problema que se encaixa na CLASSE A proposta por Lacomme \& Prins [6, 7], porém uma particularidade que o torna diferente dos propostos na literatura é que ao final de cada dia de trabalho o carro não necessita voltar para um depósito, ele permanece onde está ao final do dia e, no próximo, parte do ponto onde parou no dia anterior.

\section{Metodologia}

A malha ferroviária tem diversos pontos que poderiam ser tratados como vértices, a fim de simplificar o problema os vértices foram definidos no grafo de forma que o deslocamento entre um ponto e outro fosse baseado numa decisão operacional que corresponde exatamente à capacidade de execução de serviço de um carro em um dia. A Figura 2 ilustra um exemplo do problema:

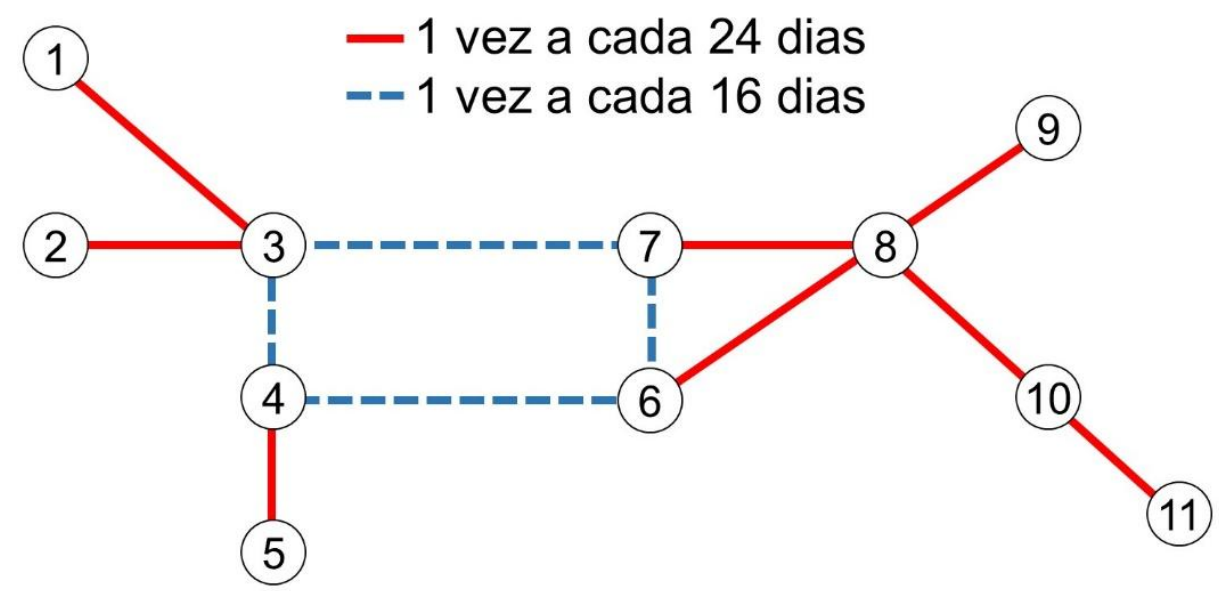

Figura 2: Exemplo do problema. Fonte: $\mathrm{O}$ autor.

Na Figura 2, um carro consegue se deslocar entre os pontos 3 e 7 durante um dia, 7 e 8 durante outro e assim por diante. Com essa simplificação, as restrições de capacidade dos carros no modelo matemático acabam implícitas em uma variável binária, onde cada carro só poderá trabalhar em uma aresta diariamente. $\mathrm{O}$ arco formado pelos pontos 4 e 6 tem uma periodicidade de 16 dias, o que significa que esse arco não pode ficar mais de 16 dias sem ser atendido por um carro. Ainda nesse exemplo, o horizonte de tempo se torna igual à maior periodicidade entre todos os arcos (24 dias) e ao final do último dia o carro deve voltar ao ponto inicial para dar início a um novo ciclo.

\subsection{Formulação Matemática}

A formulação parte de um grafo não direcionado $G=(V, A)$, com $n$ pontos, $\log o, V=\left\{v_{l}\right.$, $\left.v_{2}, \ldots, v_{n}\right\}$, e $m \operatorname{arcos} \operatorname{com} A=\left\{a_{1}, a_{2}, \ldots, a_{m}\right\}$ que devem ser percorridos por $n k$ carros definidos 
pelo conjunto $K=\{1,2, \ldots, k\}$. Cada arco é formado por um par de nós, é chamado de $x_{i j}=\left(v_{i}, v_{j}\right)$, e é associado a um custo $c_{i j}$. A capacidade de cada carro é de percorrer 1 arco por dia, e a demanda ou periodicidade de cada arco é expressa na quantidade máxima de períodos em que o arco deve ser atendido ao menos uma vez $M P\left(x_{i j}\right)$. Tem-se ainda o horizonte de tempo $H$, formado por $n p$ períodos, onde cada período é simbolizado pela letra $p$.

O modelo é baseado em Programação Linear Binária, logo para a resolução do problema foram criadas três variáveis binárias apresentadas abaixo:

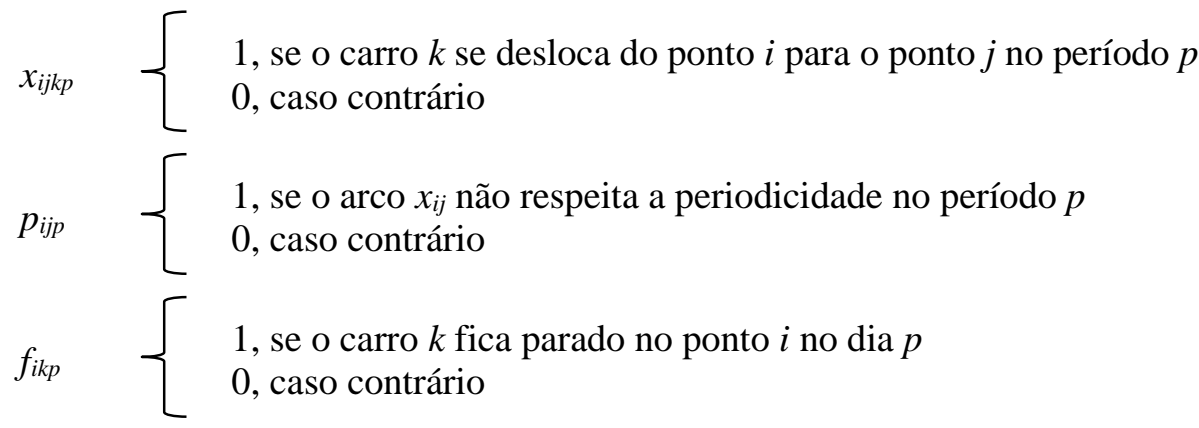

As variáveis $p_{i j p}$ e $f_{i k p}$ foram criadas para que o problema tenha uma factibilidade. Cada variável $p_{i j p}$ tem associado a ela um custo $P U_{i j}$, que é uma punição caso a periodicidade de um arco não seja atendida. E a variável $f_{i k p}$ permite que o carro folgue em um determinado dia, pois algumas vezes é possível obter uma solução com menos deslocamentos se o carro esperar para atravessar um arco.

A função objetivo é dada pela equação (1):

$$
\min z=\sum_{\left(v_{i}, v_{j}\right) \in A} \sum_{A=1}^{n k} \sum_{p=1}^{n p} c_{i j} * x_{i j k p}+\sum_{\left(v_{i}, v_{j}\right) \in A} \sum_{p=1}^{n p} P U_{i j} * p_{i j p}
$$

E os problemas estão sujeitos às seguintes restrições:

$$
\begin{aligned}
& \sum_{\left(v_{j}, v_{i}\right) \in A} x_{j i k p}+f_{i k p}-\sum_{\left(v_{i}, v_{j}\right) \in A} x_{i j k, p+1}-f_{i k, p+1}=0 \quad \forall v_{i} \in V, k, p \\
& \sum_{\left(v_{j}, v_{i}\right) \in A} x_{i j k p}+\sum_{\left(v_{j}, v_{i}\right) \in A} x_{j i k p}+\sum_{i=1}^{n} f_{i k p}=1 \quad \forall p, k \\
& \sum_{k=1}^{n k}\left(\begin{array}{c}
x_{i j k p}+x_{j i k p}+x_{i j k, p+1}+x_{j i k, p+1}+\ldots \\
+x_{i j k, p+M P\left(x_{i j}\right)}+x_{j i k, p+M P\left(x_{i j}\right)}
\end{array}\right)+p_{i j p} \geq 1 \quad \forall\left(v_{i} v_{j}\right) \in V, p \\
& x_{i j k p}, p_{i j p}, f_{i k p} \quad \in\{0,1\} \quad \forall\left(v_{i} v_{j}\right) \in V, k, p
\end{aligned}
$$

A restrição (2) garante o fluxo diário dos carros permitindo a folga, sendo que, como serão formados ciclos, ao fim do período $n p$ o carro deve retornar para o mesmo local em que estava no dia 1, ou seja, o período $n p+1$ é igual ao dia 1 , e assim por diante. Isso vale para todo o horizonte de tempo e para todas as restrições. A restrição (3) garante que todos os carros terão alguma designação para o dia $p$, além disso, garante que a capacidade de cada carro não seja excedida. Já a restrição (4) é a mais complexa, ela se refere à periodicidade em cada trecho e é quando as variáveis $p_{i j p}$ podem assumir valor 1 , ou seja, podem ocorrer punições. Contudo, se a necessidade for de apenas uma passagem durante todo o horizonte de tempo, a restrição pode ser simplificada para apenas um dia $p$ e a variável $p_{i j p}$ pode ser descartada. E, finalmente, a equação (5) determina que todas as variáveis sejam binárias. 


\section{Considerações finais}

Os problemas de roteamento em arcos têm grande aplicabilidade e podem ser adaptados conforme as necessidades. O PCARP é um problema NP-Hard e quanto mais restrições tiver, seja quanto à quantidade, capacidade, ou tempo, mais difícil é encontrar uma solução. O problema aqui proposto foi modelado e executado no CPLEX $12.4 \mathrm{em}$ um computador Core i5 com processamento de 3,1 GHz com 8 Gb de memória RAM em um sistema operacional de 64 bits. Instâncias pequenas como a da Figura 2, com apenas 1 carro, 11 pontos, 12 arestas e 2 periodicidades obtiveram solução exata em 387 segundos. Problemas mais complexos com dois carros, envolvendo um horizonte de tempo de 60 dias, 4 periodicidades, 21 pontos e 22 arcos são processados até a memória do computador se esgotar e não se obtêm uma solução ótima, porém se o programa for limitado a 24 horas de iterações consegue-se bounds com boas soluções.

Algumas adaptações que podem ser feitas decorrem da necessidade de que o carro trabalhe continuamente sem parar, neste caso não é necessário utilizar as variáveis $f_{i k p}$, ou, se não for permitido que atrasos ocorram, eliminam-se as variáveis $p_{i j p}$. Entretanto, essas adaptações podem fazer com que o problema torne-se infactível.

Para trabalhos futuros é interessante desenvolver heurísticas e comparar soluções para escolher um método apropriado para resolução do problema, além disso, podem-se desenvolver modelos para definir a real necessidade de equipamentos para malha de forma que todos os serviços sejam atendidos sem que ocorram ociosidade ou atrasos.

\section{Referências}

[1] Chu, F. et al. 2005. Heuristics for the periodic capacitated arc routing problem. Journal of Intelligent Manufacturing. 16, 2 (2005), 243-251.

[2] Eiselt, H. a. et al. 1995. Arc Routing Problems, Part II: The Rural Postman Problem. Operations Research. 43, 3 (1995), 399-414.

[3] Eiselt, H. a. et al. 1995. Arc Routing Problems, Part I: The Chinese Postman Problem. Operations Research. 43, 2 (Mar. 1995), 231-242.

[4] Ghiani, G. et al. 2005. A heuristic for the periodic rural postman problem. Computers \& Operations Research. 32, 2 (Feb. 2005), 219-228.

[5] Hashemi Doulabi, S.H. and Seifi, A. 2013. Lower and upper bounds for location-arc routing problems with vehicle capacity constraints. European Journal of Operational Research. 224, 1 (Jan. 2013), 189-208.

[6] Lacomme, P. et al. 2002. Evolutionary Algorithms for Multiperiod Arc Routing Problems, em Proc. of the 9th Int. Conf. on Information Processing and Management of Uncertainty in Knowledge-Based systems, IPMU 2002, 01-05/07/02, Annecy, France, ESIAUniversity of Savoie. pp. 845-852.

[7] Lacomme, P. and Prins, C. 2005. Evolutionary algorithms for periodic arc routing problems. European Journal of Operational Research. 165, (2005), 535-553.

[8] Monroy, I.M. et al. 2013. The periodic capacitated arc routing problem with irregular services. Discrete Applied Mathematics. 161, (2013), 691-701. 